\title{
ECG and echocardiographic diagnosis of pulmonary thromboembolism associated with central venous lines
}

Andrew J Pollard, Narayanswami Sreeram, John G Wright, Susan V Beath, Ian W Booth, Deirdre A Kelly

\section{Abstract}

The aim was to establish the prevalence of pulmonary embolism in 21 children (median age 12 months; range 5-132 months) with central venous lines in situ $>3$ months (median 10 months; range 3-47). Twelve-lead electrocardiograms (ECGs) and echocardiograms were analysed in a retrospective study using ECG and echocardiographic criteria for pulmonary embolism - previously established and validated in adult patients and standard paediatric ECG values as control data. Patients were scored as having definite $(n=7)$, probable $(n=5)$, or $57 \%$ of ECGs showed abnormalities compatible with pulmonary embolism. In two patients, serial ECGs obtained during an acute cardiorespiratory illness showed cumulative changes diagnostic of pulmonary embolism. Eight of 12 patients with abnormal ECGs had echocardiography; in seven of these $(88 \%)$ the right ventricular end diastolic diameter was $>$ 2SD above the mean value for age. Twelve of the patients included in this study have died; two died following an acute respiratory illness. There was postmortem evidence of pulmonary thromboembolism in both of the two children for whom necropsy information was available. The data suggest that pulmonary embolism is common in children who have central venous lines in situ for $>3$ months. Serial studies are of value in some patients. Pulmonary embolism may compromise the long term survival of children with small bowel failure and preclude consideration for liver and small bowel transplantation.

(Arch Dis Child 1995; 73: 147-150)

Ladywood Middleway, Birmingham B16 8ET

The Liver Unit

A J Pollard

D A Kelly

The Heart Unit

N Sreeram

J G Wright

Department of

Paediatric

Gastroenterology

S V Beath

I W Booth

Correspondence to:

Dr D A Kelly.

Accepted 18 April 1995 Keywords: pulmonary embolism, central venous lines, parenteral nutrition, electrocardiogram.

Increasing numbers of children receive parenteral nutrition for a variety of indications, and some will be candidates for small bowel transplantation. ${ }^{1}$ The hypertonic solutions infused necessitate access to a large central vein, and the complications of sepsis and hepatic dysfunction are well recognised. ${ }^{2}$ Until recently, pulmonary embolism was thought to be uncommon in these patients, but there is increasing concern that recurrent microemboli no pulmonary embolism $(n=9)$. Overall

may compromise cardiopulmonary function and thereby preclude transplantation. ${ }^{34}$

The sensitivity of ventilation-perfusion lung scintigraphy in the diagnosis of major pulmonary embolism is low. ${ }^{5}$ Although the more invasive technique of pulmonary angiography is more sensitive in the diagnosis of embolism to larger pulmonary arteries, ${ }^{6}$ the usefulness of these two investigations is likely to be limited in chronic or recurrent embolus of microthrombi. In this study we aimed to document electrocardiographic and echocardiographic evidence for recurrent or chronic pulmonary thromboembolism in children with long term central venous lines.

\section{Methods}

A retrospective study was performed between 1988-1994 using the databases from the liver unit, the department of paediatric gastroenterology, and the pharmacy parenteral nutrition database at Birmingham Children's Hospital. Twenty one children (12 male, nine female) who had central venous lines in situ for more than three months (median 10 months; range 3-47) and who had undergone formal cardiac assessment were identified. Their median age was 12 months (range 5-132 months).

Fourteen children had short gut syndrome (gastroschisis, 8; intestinal atresia, 2; necrotising enterocolitis, 3; volvulus, 1), two had hollow visceral myopathy, two had idiopathic intractable diarrhoea of infancy (antienterocyte autoantibody negative), one had autoimmune enteropathy, and one had received a bone marrow transplant for acute leukaemia. The remaining child had a liver and small bowel transplant for gastroschisis with short gut syndrome and parenteral nutrition associated liver disease.

Standard paediatric parenteral nutrition regimes were used ${ }^{7}$ with adjustments made in response to biochemical and anthropometric information. No heparin was added to the parenteral nutrition and no patient received systemic anticoagulation. In this retrospective study we do not have coagulation profiles paired with the electrocardiograms (ECGs) or echocardiograms of our patients. None of our patients had a known thrombotic tendency or was anticoagulated.

Standard 12-lead ECGs were performed as part of an assessment protocol for liver or liver and small bowel transplantation in nine children and as a diagnostic procedure in nine 
Frequency of positive ECG criteria for pulmonary tromboembolism (PE) in all patients

\begin{tabular}{llll}
\hline Criterion & Definite PE $(n=7)$ & Probable PE $(n=5)$ & No PE $(n=9)$ \\
\hline S $>1 \cdot 5$ mm in I and aVL or R/S $<1$ & 7 & 4 & 5 \\
Q in III and aVF, but not II & 5 & 4 & 4 \\
Negative T in III, aVF, V1-V5 & 5 & 2 & 1 \\
Right bundle branch block & 3 & 0 & 1 \\
ST elevation in V1 & 1 & 0 & 1 \\
Upright T waves in V1 & 0 & 1 & 0 \\
QRS <5 mm in all limb leads & 3 & 1 & 1 \\
Transition in V5 or V6 & 4 & 0 & 0 \\
Frontal QRS >90 & 1 & 0 & 0 \\
Indeterminate axis & 3 & 1 & 0 \\
Right atrial hypertrophy & 0 & 1 & 0 \\
Atrial arrhythmia & 0 & 1 &
\end{tabular}

Patients were defined as having definite PE if four or more of the ECG criteria were positive, probable $P E$ (three positive criteria) or no $P E(0-2$ positive criteria).

children who had suffered an acute cardiorespiratory illness. In three children the ECGs were performed as part of the investigation of an associated systolic murmur. ECGs and echocardiograms, where available, were examined retrospectively for each patient.

The ECG criteria listed in the table, previously established and validated in adult patients using ventilation/perfusion scans or pulmonary angiography, ${ }^{8}$ were used to diagnose pulmonary embolism. All ECGs were examined retrospectively by the same observer - who did not have clinical information about the patient - for right ventricular strain in particular, using 12 criteria, including the conventional ' $S_{I} Q_{\text {III }} T_{\text {III }}$ ' by comparison with standard values. Patients were scored as having definite pulmonary embolism (four or more positive criteria); probable pulmonary embolism (three criteria), or no pulmonary embolism (two or less positive criteria). Cumulative changes on serial ECGs were only accepted if they developed during the same acute cardiorespiratory illness.

Echocardiograms, when available, were reviewed and the following criteria sought ${ }^{9}{ }^{10}$ : (1) right ventricular internal diameter measured in the four-chamber view at the level of the tips of the leaflets of the tricuspid valve, perpendicular to the long axis of the ventricle, at the onset of the $R$ wave of the ECG (average of three measurements); (2) interventricular septal motion in the short axis view reported as

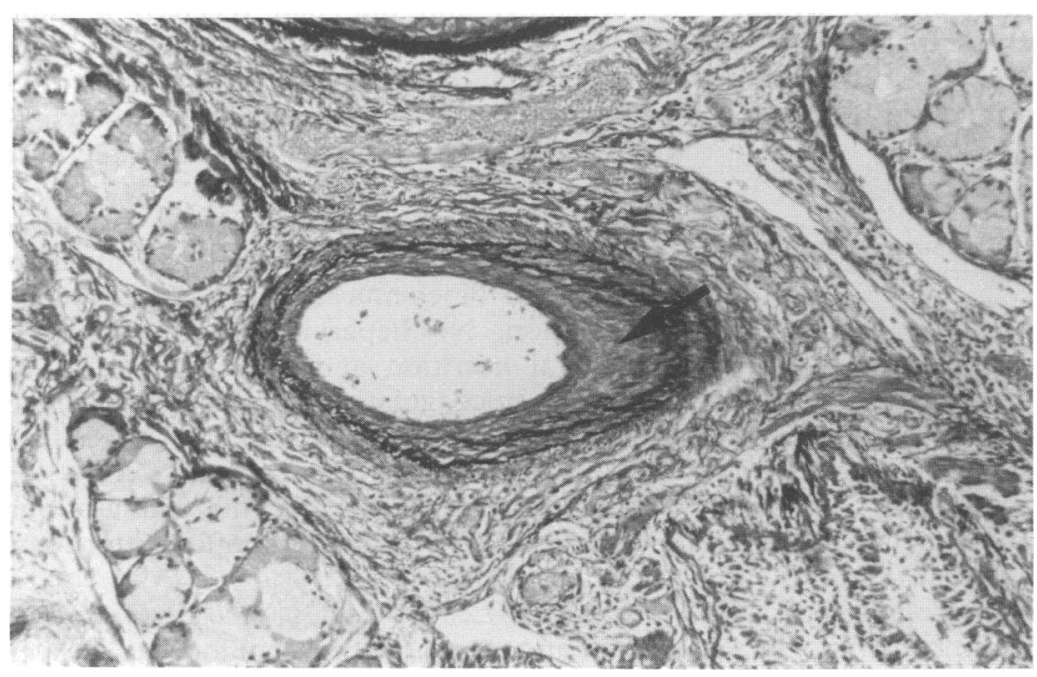

Necropsy section of lung showing eccentric intimal thickening (arrow) in a pulmonary vessel. This child with a central venous line died following a respiratory illness. normal, flattened, or bulging towards the left ventricle in diastole; (3) the peak velocity of the tricuspid regurgitant jet (TR), where present, as an estimate of pulmonary arterial pressure. ${ }^{11}$

Two children had necropsy examinations which were reviewed for description of pulmonary thromboembolism or catheter associated thrombus.

\section{Results}

Using the criteria described, seven patients were scored as having definite pulmonary embolism, five had probable pulmonary embolism, and nine had no pulmonary embolism. The table lists the frequency with which individual ECG criteria occurred in the 21 patients studied. Overall $12 / 21(57 \%)$ of ECGs showed abnormalities compatible with pulmonary embolism. In two patients, serial ECGs obtained during an acute cardiorespiratory illness showed cumulative changes diagnostic of pulmonary embolism.

Eight of 12 patients with abnormal ECGs had undergone echocardiography; in seven of these $(88 \%)$ the right ventricular end diastolic diameter was $>2$ SD above the mean value for age. None of the patients had abnormalities of interventricular septal motion, or identifiable tricuspid valve regurgitation on Doppler colour flow imaging. Two patients had intraatrial thrombi on echocardiography.

Twelve of the patients in this study have died. In the two children who underwent necropsy examination there was evidence of pulmonary thromboembolism. The first patient died aged 3 years from respiratory failure, six months after liver and small bowel transplantation, which had been undertaken for short gut syndrome and liver dysfunction associated with prolonged use of parenteral nutrition following gastroschisis. Necropsy examination of the lungs showed multiple lipid emboli, pulmonary vascular muscular hypertrophy (consistent with mild pulmonary hypertension), multiple fibrin thrombi, and eccentric intimal proliferation in large blood vessels (see figure). These features indicate recurrent pulmonary thromboembolism. The second patient died at the age of 3.9 years from multiorgan failure secondary to autoimmune enteropathy and cirrhosis following parenteral nutrition. At necropsy there were calcified emboli in the pulmonary capillaries and arteries. Both patients had ECG criteria compatible with pulmonary embolism.

\section{Discussion}

The use of central venous lines in children is widespread but in most instances for a few weeks only. Recent reports have recognised that septic, thrombotic, and catheter fragment emboli are complications of central venous line insertion. ${ }^{341213}$ Platelet aggregates are a common finding in the small lung vessels of children dying during liver transplantation and the presence of intravascular catheters may be contributory. ${ }^{14}$ However, the incidence 
and significance of pulmonary embolism in children receiving long term parenteral nutrition still needs to be defined, as this may adversely affect further management.

Acute pulmonary embolism is difficult to recognise clinically in infants and children, in whom the clinical picture may be more suggestive of pneumonia. Dyspnoea is a common symptom, but pain and collapse do not usually occur. ${ }^{15}$ Respiratory symptoms are frequently reported by the parents of children with long term indwelling central venous lines but their relation to pulmonary embolism is unknown. In a recent report, major venous thrombosis was visualised by echocardiography in 12 of 34 children with central venous lines for parenteral nutrition. Three of the four deaths in this group were attributed to pulmonary embolism. ${ }^{16}$

Several invasive and semi-invasive methods are available for diagnosing acute pulmonary embolism. However, the sensitivity of ventilation-perfusion lung scintigraphy in the diagnosis of major pulmonary embolism is low, even in patients with 'high probability' scans, ${ }^{5}$ and its usefulness is likely to be limited in chronic or recurrent embolus of microthrombi. Pulmonary angiography is more sensitive than lung scinitigraphy in the diagnosis of embolism $^{6}$ in larger pulmonary arteries but it is an expensive invasive investigation that carries a small but definite risk of serious complications. Neither of these techniques has been used extensively in children.

Electrocardiography and echocardiography are valuable techniques for diagnosing pulmonary embolism in adults, but have not been systematically evaluated in children. We have recently shown that the presence of three or more of the ECG criteria described above were diagnostic of pulmonary embolism in adults ${ }^{8}$ and demonstrated the increased sensitivity of multiple criteria in diagnosing pulmonary embolism, as compared with the classical criteria originally proposed by McGinn and White. ${ }^{17}$ None of the new criteria taken alone has a greater predictive value for diagnosis of pulmonary embolism than any other criterion. In the absence of established ECG criteria for pulmonary embolism in children, we have applied the same measures. As neonates and young infants may have a frontal QRS axis of $>90^{\circ}$ on ECG, we have only used this criterion for patients over one year of age. We have also been more stringent in the application of these criteria, by defining definite pulmonary embolism as occurring only if four or more, rather than three or more, were satisfied.

Echocardiographic diagnosis of pulmonary embolism depends on direct imaging of pulmonary arterial thrombi. ${ }^{9}$ As echocardiography is only capable of evaluating the central pulmonary arteries, indirect indices of increased right ventricular volume or pressure have been used to establish the diagnosis, and have been shown to be very sensitive in adults. ${ }^{9} 10$ Some of these criteria have not been validated in children. However, the finding of a significantly increased right ventricular end diastolic diameter in seven out of eight patients with ECG evidence of pulmonary embolism provides excellent additional evidence for right ventricular volume overload in these patients. In the absence of structural cardiac defects or a previous history of cardiovascular disease, these findings may be attributable to pulmonary embolism. The absence of measurable tricuspid valve regurgitation or diastolic bulging of the interventricular septum to the left suggest either that children behave differently when compared with adults, or that recurrent small emboli may be occurring in these patients, who do not therefore show evidence of acute pressure overload of the right ventricle.

This study reports the finding that $57 \%$ of children who had central venous lines for parenteral nutrition for three or more months have ECG evidence of pulmonary embolism. In a recent study of 34 children receiving long term parenteral nutrition, Dollery et al found that up to one third had major venous thrombosis by echocardiography and isotope lung scanning, with a $29 \%$ prevalence of pulmonary embolism. ${ }^{16}$ Previous studies have suggested that the prevalence is $5 \%$ or less. ${ }^{1819}$ Our data suggest that the true prevalence of pulmonary embolism is higher than this. It is possible that this difference reflects the sensitivity of our technique in identifying smaller, more peripheral microemboli, in the absence of major thrombus. Indeed, we saw only two intra-atrial thrombi on reviewing the echocardiograms. Moreover, Dollery's study included 15 patients with autoimmune enteropathy who seemed more susceptible to thrombosis. We had only one patient with autoimmune enteropathy and still found an overall higher prevalence of pulmonary embolism.

There was no relation in our patients between diagnostic group or duration of parenteral nutrition and pulmonary embolism in this retrospective study. In Dollery's study, the number of central venous line changes correlated with a decreased risk of embolism. ${ }^{16}$ We could not show this.

There was postmortem evidence of pulmonary embolism in both of the children in our study who had necropsies. In adults dying from all causes, venous thromboses and pulmonary embolism are frequent necropsy findings, ${ }^{20}$ and the necropsy may be misleading as a tool for assessing risk of pulmonary embolism from central venous catheters in the adult. The situation is different in children, as there were only eight cases $(0.05 \%)$ of massive pulmonary thromboembolism found in a 50 year retrospective review of 17500 paediatric necropsies. ${ }^{21}$

We conclude that children who have long term indwelling central venous lines are at risk of pulmonary embolism which may exclude them from small bowel and liver transplantation. Future management of these children should include strategies to reduce the risk of clots forming on the catheters (for example, use of heparin, regular flushing with thrombolytic agents, prophylaxis with aspirin), and to identify and remove thrombi promptly. Prospective studies are required to assess 
whether thrombolysis may be beneficial in selected cases presenting with acute cardiorespiratory symptoms.

We would like to thank Patrick Ball for providing access to the Children's Hospital; to Dr P Darbyshire for permission to include one of his patients; to the paediatric su our lines, in particular Mr R Buick, Mr S Corkery and $\mathbf{M r} \mathbf{P}$ Gornall; and to the secretaries and ECG technicians in the heart unit at The Children's Hospital, Birmingham for providing the echo- and electrocardiographic data.

1 Beath SV, McKiernan PJ, Murphy MS, et al. Paediatric referrals for combined liver and small bowel transplantation: timing and indications - room for improvement? (abstr) Proc Nutr Soc 1994; 53: 48A.

2 De Potter S, Goulet O, Lamor M, et al. 263 patient years of home parenteral nutrition in children. Transplant Proc 1992; 24: 1056-7.

3 Rello J. Pulmonary embolism due to an indwelling central venous catheter. Chest 1991; 100: 1477-8.

4 Shiba E, Kambayashi J, Sakon M, Yoshida T, Arisawa J, Mori T. Septic pulmonary emboli after prolonged use of central venous catheter for parenteral nutrition. Eur $\mathcal{F}$ Surg central venous cathet

5 The PIOPED Investigators. Value of the ventilation/perfusion scan in acute pulmonary embolism: results of the prospective investigation of pulmonary embolism diagnosis (PIOPED). $\mathscr{F} A M A$ 1990; 263: 2753-9.

6 Marsh JD, Glynn M, Torman HA. Pulmonary angiography. Application in a new spectrum of patients. Am $\mathcal{J}$ Med 1983; 75: 763-70.

7 Ball PA, Booth IW. Parenteral nutrition. In: Insley J, ed. A paediatric vade-mecum. 1990; 2: 22-8.

8 Sreeram N, Cheriex EC, Smeets JLRM, Gorgels AP, Wellens HJ. Value of the 12-lead ECG at hospital admission in the diagnosis of pulmonary embolism. $A m \mathcal{F}$ Cardiol 1994; 73: 298-303.

9 Cheriex EC, Sreeram N, Eussen YFJM, Pieters FAA, Wellens HJJ. Cross sectional Doppler echocardiography as the initial technique for the diagnosis of acute pulmonary embolism. Br Heart f 1994; 72: 52-7.

10 Sreeram N, Cheriex E, Eussen Y, Pieters FA, Wellens HJ. Two-dimensional Doppler echocardiography as the initial
diagnostic tool for acute pulmonary embolism. (abstr) $\mathcal{f}$ Am Coll Cardiol 1994; 23: 95A.

11 Yock PG, Popp RL. Noninvasive estimation of right ventricular systolic pressure by Doppler ultrasound in ventricular systolic pressure by Doppler ultrasound in patients

12 Johnson CW, Miller DL, Ognibene FP. Acute pulmonary embolism associated with guidewire change of a central venous catheter. Intensive Care Med 1991; 17: 115-7.

13 Polos PG, Sahn SA. Complication of central venous catheter insertion: fragmentation of a guidewire with pulmonary artery embolism. Crit Care Med 1991; 19: 438-40.

14 Gosseye S, van Obbergh L, Weynand B, et al. Platelet aggregates in small lung vessels and death during liver transplantation. Lancet 1991; 338: 532-4.

15 Stern RC. Pulmonary embolism and infarction. The respiratory system. In: Behrman RE, ed. Nelson textbook of pediatrics, 14th ed. Philadelphia: WB Saunders Co, 1992: pediatrics,

16 Dollery CM, Sullivan ID, Bauraind O, Bull C, Milla PJ. Thrombosis and embolism in long-term central venous Thrombosis and embolism in long-term central venous

17 McGinn S, White PD. Acute cor pulmonale resulting from pulmonary embolism. $\{A M A$ 1935; 104: 1473-80.

18 Schmidt-Sommerism. FAMA 1935 ; 104: 1473-8. Catheter-related complications in 35 children and Catheter-related complications in 35 children and nutrition. F Parenter Enter Nutr 1990; 14: 148-51.

19 Leiby JM, Purcell H, De Maria J, Kraut EH, Sagone AL, Metz EN. Pulmonary embolism as a result of Hickman catheter-related thrombosis. Am f Med 1989; 86: 228-31.

20 Diebold J, Lohrs U. Venous thrombosis and pulmonary embolism. A study of 5039 autopsies. Path Res Pract 1991; 187: 260-6.

21 Byard RW, Cutz E. Sudden and unexpected death in infancy and childhood due to pulmonary thromboembolism. An autopsy study. Arch Path Lab Med 1990; 114: $142-4$. 